\title{
A New Robust Optimization Approach to Deal with Dependent Uncertain Parameters
}

\author{
Amirhossein Mostofi \\ School of Management \\ Victoria university of Wellington \\ Wellington, New Zealand \\ e-mail: Amirhossein.mostofi@vuw.ac.nz
}

\author{
Vipul Jian \\ School of Management \\ Victoria university of Wellington \\ Wellington, New Zealand \\ e-mail: Vipul.jain@vuw.ac.nz
}

\author{
Mojtaba Arab Momeni \\ Department of industrial Engineering \\ Iran university of Science and Technology \\ e-mail: M arabmomeni@ind.iust.ac.ir
}

\begin{abstract}
In the optimization problems with uncertain parameters, a solution is said to be robust if it is feasible with high probability regarding the realization of uncertain parameters. In this paper, a new robust approach is developed for the linear problems in which the model parameters are dependent on each other. The proposed approach converts the linear model to an equivalent integer linear programming one using the primal and dual theorem. The results of the paper indicate the ability of the new approach in fixing some inconsistency of the common robust optimization approach for the mentioned problem.
\end{abstract}

Keywords-deterministic robust optimization; dependent uncertain parameters; consistence robust solution; primal and dual relations

\section{INTRODUCTION}

The robust optimization approaches encompass techniques and methodologies that support decision-making against parameter uncertainty to find a robust solution [1].In the concept of robustness, a range of solutions should be considered, which are obtained by taking different possible values of uncertain parameters. On one hand of this range, there is a thoroughly conservative solution that remains feasible for any realization of the uncertain parameters as a "pessimistic solution." At the opposite end, there is an "optimistic solution," i.e., the solution which could be obtained if the uncertain parameters are realized in the state wherein we have the best value of the objective function. In reality, the conservative approach is appropriate for engineering applications such as robust control theory; for example, a doomed satellite launch or a destroyed unmanned robot results in a significant adverse effect that cannot be connived. However, in business practices, the argumentative event, such as low demand, does not lead to high consequences as an engineering practice. Business managers usually seek a feasible solution with a high level of confidence and also high value for a considered objective function. Indeed, they are not interested in complete protection against uncertain events at the expense of rigorous deterioration in the objective function [1]. Therefore, the solutions which lie on a spectrum between these two extremes may be more desirable for decision-makers. Each of the solutions in this range can be regarded as a robust solution with a different degree of robustness.

In reality, the uncertainty in the model parameters comes from different resources such as the uncertainty of system outputs related to approximations in the modeling process [2, 3], production tolerances and actuator imprecision intentionally exposed for being the model simple and cost and time effective and changes in environmental and operating conditions of the model in the future that could not be predicted precisely before that $[4,5]$.

In mathematical optimization, the uncertainty in the objective function and the feasible region of solutions are sometimes distinguished. When the uncertainty only influences the feasibility of solutions, then robust optimization approaches aim to seek a feasible solution for any realization of unknown coefficient within a smaller realistic set, which is called "uncertainty set." This set sometimes is centered on the nominal values of uncertain parameters. The choice of uncertainty set plays an important role in determining the level of deterioration in the objective function and the level of protection against uncertainty and feasibility of the resulting solutions [1, 6, 7]. When the uncertainty exists in parameters of an objective function, the robust optimization approach pursues a solution for any realization of unknown parameters. In this case, a common approach is to optimize the worst-case of the objective function. Also, this can be done by incorporating the objective function as a new constraint into the model constraints. Moreover, the researchers examined both single objective function[8,9] and multi-objective function[10, 11] for achieving robust solutions. In the proposed robust approach, a worst-case analysis of objective functions has been considered. 


\section{LITERATURE REVIEW}

The duality has played a key role in the development of robust models $[12,13]$. Some researchers have investigated the relationship between primal and corresponding dual of robust optimization problems. For example, it has been shown that the worst of primal is equivalent to the dual best [14]. In the proposed robust approach, this relationship is utilized to convert a MinMax combinatorial problem to an equivalent MILP.

It is worth to note that the robust concepts and techniques have originally rooted in engineering practices, and as a preliminary effort in this field, the "Taguchi method" is a well-known practice that considers the robustness in the design of products[15]. In the operation research context, the research of [16] could be referred to as pioneering publications.

To deal with uncertainty in the robust optimization approaches, generally, three main categories exist in the literature: deterministic, probabilistic, and possibilistic. In the deterministic type, the domain of uncertain parameters is considered. This type of uncertainty arises when there is no more information about uncertain parameters except their domain of variation [12, 17].In the Probabilistic robust optimization, the likelihood of uncertain events is considered, and the probabilistic theory is extended to the robust optimization problem. In this field, the connections of the robust optimization with moment information [18, 19], twostage optimization approach [20, 21], multi-stage optimization approach [22, 23], and risk theory [24-26] have been studied. Moreover, the degree of an uncertain event is measured using fuzzy measures in the possibilistic type [27]. It is worth mentioning that in this paper, the deterministic type of uncertainty is studied, and the dependency between uncertain parameters is taken into account for avoiding inconsistency in the model results.

To measure the robustness of a solution, some robustness measures have been introduced in the literature. The robust counterpart is a robustness measure that explores a solution amongst feasible solutions with the best objective function for all values of uncertain parameters [28]. This measure is used for deterministic uncertainty type as a conservative approach. On the other hand, expectancy measures are another robustness measure that takes into account a probabilistic function of uncertain model and obtains a robust solution by optimizing this function. Conditional expected value [29] and variance [30] are examples of these measures. Another measure is the probabilistic threshold of robustness, which considers the probability distribution of an uncertain model for searching a robust solution within a predefined threshold [29]. Statistical feasibility robustness is another measure of robustness that explores a feasible solution with a predetermined probability. The chance constraint programming method is an example of this robustness measure [31]. Finally, the possibilistic uncertainty measure takes into account uncertainty that is identified subjectively. For this type of uncertainty, membership function is usually defined using a fuzzy set theory [27].
Generally, to implement the ideas of the robustness, two approaches exist. One approach is the "simplification strategy" introduced by [32], whereas another approach is "simulation optimization technique" nominated by [33]. The first approach is used for modeling problems mathematically, and it is applicable if the probabilistic distribution or plausible membership function of an uncertain model can be determined or estimated. On the other hand, techniques such as Monte-Carlo simulation are applied to simulate the realworld problem in the second approach. The latter is helpful for some problems that an explicit mathematical programming model cannot model either, or their mathematical models are complicated enough to be implemented efficiency by the available standard procedures. In this paper, a linear problem with shared uncertain parameters is addressed, and a mixed-integer linear programming (MILP) model is proposed for taking into account the concept of robustness. Thus, the proposed model in this study is categorized as a "simplification strategy."

\section{STUDY DESIGN}

In this paper, it is assumed that uncertain events are common between the parameters of the model. Therefore, it is not reasonable to consider these parameters individually. Here, each event is defined as a random variable, whereas only its domain is known. The models introduced by [17],[34], and [12] are among the robust models which regard the domain of uncertain parameters. [17] developed a robust model under the uncertain condition with a fully conservative robust approach, wherein this solution remains feasible for all realization of uncertain parameters. The advantage of the Soyster approach is that a linear programming model remains linear after applying robustness modifications. However, that conservative and worst-case analysis approach does not yield good value for the objective function of the problem. [34] proposed a less conservative robust model that made a tradeoff between feasibility and optimality of the solutions. Indeed, that method converted a linear programming problem to a nonlinear one and made it more challenging to solve. One of the applicable robust optimization approaches is [12] that has been great fund interest between researchers and practitioners. Moreover, this approach has the ability to doing tradeoff efficiently between feasibility and optimality of solutions. However, that approach does not consider repetitive uncertain parameters. Thus, we utilized the idea of robustness in [12] and developed a new robust optimization approach for the problem with dependent uncertain parameters.

The rest of this paper is organized as follows. First, after a brief description of the [12] approach, the challenges of this approach are discussed. Then, the new robust approach, which overcomes the mentioned shortcomings of the [12] is developed. After that, numerical examples are conducted to show the validation of the proposed approach. A discussion section follows these lines. Finally, the results of the paper and future research opportunities are presented. 


\section{ASSUMPTIONS AND ANALYSIS}

\section{A. A Model with Shared Parameters}

Before explaining the proposed robust optimization approach, we first consider a simple production planning model. Also, we show some inconsistency in the robust solution of the model based on [12] approach. This model is as follow:

Problem (1)

$$
\begin{aligned}
& \operatorname{Max} c_{1} d_{1} x_{1}+c_{2} d_{2} x_{2}+c_{3} d_{3} x_{3}+c_{4} d_{4} x_{4} \\
& \text { s.t. } \\
& \quad a_{1} d_{1} x_{1}+a_{2} \cdot d_{2} x_{2}+a_{3} d_{3} x_{3}+a_{4} d_{4} x_{4} \leq C \\
& \quad \forall i, \quad 0 \leq x_{i} \leq 1
\end{aligned}
$$

The model aims to maximize the net profit of production in a firm with total production capacity equal to $C$. Here, $d_{\mathrm{i}}$ $(\mathrm{i}=1,2,3,4)$ stands for the uncertain demand of product $i$. Moreover, $x_{i}$ denotes the percentage of product $i$ demand, which is prepared by the firm as the model variable. Also, $c_{i}$ and $a_{i}$ are the net profit per unit and the capacity usage per unit for the product $i$. In the following, after explaining [12] approach, we show how this approach results in an incompatible robust solution for the production planning problem. [12] approach considers a linear programming model as follow:

$\operatorname{Max} C x$

s.t.

$$
\begin{aligned}
& A x \leq b \\
& l \leq x \leq u
\end{aligned}
$$

In this model, it is assumed that $J_{i}$ is the set of coefficients in row $i$ of matrix $A$ that are subject to uncertainty. Each entry $a_{i j} \mid j \in J_{i}$ in row $i$ is a symmetric and bounded random variable that takes values in an interval $\left[a_{i j}-\hat{a}_{i j}, a_{i j}+\hat{a}_{i j}\right]$. Associated with the uncertain variable $a_{i j}$, the random variable $\eta_{i j}=\left(a_{i j}-a_{i j}\right) / a_{i j}$ is regarded, which is assumed to follow an unknown but symmetric distribution in the interval $[-1,1]$. Following the above assumptions and denoting $\Gamma_{i}$ as the desired number of uncertain parameters in row $i$ which are regarded as critical parameters, [12] introduced the equivalent robust model of the above linear model as follow:

$\operatorname{Max} C^{\prime} X$

$$
\begin{aligned}
& \quad s . t . \\
& \sum_{j} a_{i j} x_{j}+ \\
& \max _{S_{i}}\left\{\sum_{j \in s_{i}} \hat{a}_{i j} y_{i}+\left(\Gamma_{i}-\left\lfloor\Gamma_{i}\right\rfloor\right) \hat{a}_{i t_{i}} y_{t}\right\} \leq b_{i} \quad \forall i \\
& S_{i}=\left\{s_{i} \cup\left\{t_{i}\left|s_{i} \subseteq J_{i},\right| s_{i} \mid=\left\lfloor\Gamma_{i}\right\rfloor, t_{i} \in J_{i} \backslash s_{i}\right\}\right. \\
& -y_{j} \leq x_{j} \leq y_{j} \\
& L \leq X \leq U \\
& Y \geq 0
\end{aligned}
$$

Also, using the primal and dual relations, [12] showed that the above robust model is equivalent to the linear model as below:

$$
\begin{array}{ll}
\operatorname{Max} & C^{\prime} X \\
& \\
& \\
\sum_{j} a_{i j} x_{j}+Z_{i} \Gamma_{i}+\sum_{j \in J_{i}} P_{i j} \leq b_{i} \quad \forall i \\
Z_{i}+P_{i j} \geq \hat{a}_{i j} y_{j} \quad \forall i, j \in J_{i} \\
-y_{j} \leq x_{j} \leq y_{j} & \forall j \\
L \leq X \leq U & \\
P_{i j} \geq 0 & \forall i, j \\
Z_{i} \geq 0 & \forall i \\
y_{j} \geq 0 & \forall j
\end{array}
$$

The idea behind this model is to choose $\Gamma_{i}$ uncertain parameters in the constraint $i$ and set their values equal to their upper or lower bounds such that the constraint imposes the most restriction on the feasible space of solutions. In this way, the feasibility of the obtained solution is ensured with more probability than a thoroughly conservative approach.

Notably, the variable $P_{i j}$ in the constraints (13) and (14) denotes whether parameter $j$ in constraint $i$ take its upper bound or lower bound as a critical value or its mean value. In this regard, using the dual-primal relations for developing this model as introduced in [12], if $P_{i j}$ is positive in the obtained robust solution, then this means that the parameter $j$ of constraint $i$ takes a critical value.

Considering the above discussion, we come back to Problem (1) and explain the arisen inconsistency of [12] approach for this problem. Let the value of parameters in Problem (1) is as Table I. Furthermore, we assume capacity $=90$. Moreover, to convert Problem (1) in the form of [12] model, we define the parameters $e_{i}=c_{i} . d_{i}$ and $f_{i}=a_{i} . d_{i}$ and substitute them in Problem (1). Doing so and using the interval multiplier operation, the values of $e_{\mathrm{i}}$ and $f_{\mathrm{i}}$ for different products will be as Table II.

TABLE I. Value of the Parameters in Problem (1)

\begin{tabular}{|c|llll|}
\hline $\boldsymbol{i}$ & $\mathbf{1}$ & $\mathbf{2}$ & $\mathbf{3}$ & $\mathbf{4}$ \\
\hline $\boldsymbol{a}_{\boldsymbol{i}}$ & 4 & 3 & 2 & 3 \\
$\boldsymbol{c}_{\boldsymbol{i}}$ & 8 & 4 & 3 & 4 \\
$\boldsymbol{d}_{\boldsymbol{i}}$ & 8 & 7 & 8 & 9 \\
$\hat{\boldsymbol{d}}_{\boldsymbol{i}}$ & 3 & 2 & 4 & 4 \\
\hline
\end{tabular}

Table II. Value of the Modified Parameters in Problem (1)

\begin{tabular}{|c|cccc|}
\hline $\mathbf{i}$ & $\mathbf{1}$ & $\mathbf{2}$ & $\mathbf{3}$ & $\mathbf{4}$ \\
\hline $\boldsymbol{e}_{\boldsymbol{i}}$ & 40 & 28 & 24 & 36 \\
$\hat{\boldsymbol{e}}_{\tilde{i}}$ & 15 & 8 & 12 & 16 \\
$\boldsymbol{f}_{\boldsymbol{i}}$ & 32 & 21 & 16 & 27 \\
$\hat{\boldsymbol{f}}_{\tilde{\mathbf{i}}}$ & 12 & 6 & 8 & 12 \\
\hline
\end{tabular}


Now, to model Problem (1) using [12] approach, two other modifications are done: the objective function is shifted to the problem constraints as $z \geq c_{1} d_{1} x_{1}+c_{2} \cdot d_{2} x_{2}+c_{3} d_{3} x_{3}+c_{4} d_{4} x_{4}$; the objective function of the problem is considered $\operatorname{Max} z$. Now, if Problem (1) is solved using [12] approach, the robust solution of the problem $\Gamma_{1}=2$ (the converted objective function constraint) and $\Gamma_{2}=2$ (the capacity constraint) is as Table III.

TABLE III. The Robust Solution of Problem (1) USING Bertsimas AND SIM (2004) APPROACH

\begin{tabular}{|c|cccc|}
\hline $\boldsymbol{i}$ & $\mathbf{1}$ & $\mathbf{2}$ & $\mathbf{3}$ & $\mathbf{4}$ \\
\hline $\boldsymbol{x}_{\boldsymbol{i}}$ & 0.532 & 1 & 0.665 & 1 \\
$\boldsymbol{y}_{\boldsymbol{i}}$ & 0.532 & 1 & 0.665 & 1 \\
$\boldsymbol{p}_{1 i}$ & 0 & 0.025 & 0 & 2.658 \\
$\boldsymbol{p}_{2 i}$ & 0.38 & 0 & 0 & 1.975 \\
\hline
\end{tabular}

Taking into account the value of $p_{1 i} \mid \mathrm{i}=1,2,3,4$ in Table (3), it is clear that the parameters $e_{2}$ and $e_{4}$ take their upper bound value in the optimal robust solution. This is corresponding to considering the lower bound values for $d_{2}$ and $d_{4}$. However, the parameters $e_{1}$ and $e_{3}$ take their mean value, and that is corresponding to setting $d_{1}$ and $d_{2}$ equal to their mean value. Similarly, the values of $p_{2 i} \mid \mathrm{i}=1,2,3,4$

Table (3) denote that the parameters $f_{l}$ and $f_{4}$ take their upper bound value, which is corresponding to $d_{1}$ and $d_{4}$ at their upper bounds. Subsequently, $f_{2}$ and $f_{3}$ appear with their mean value, which means $d_{2}$ and $d_{3}$ takes their mean values in the second constraint. These results show an inconsistency or a conflict in the robust solution because $d_{i}$ 's appear with different values in the different constraints.

In the next section, a new robust optimization approach is proposed, which fixed the mentioned inconsistency, although it inherits the concept of robustness addressed by [12].

\section{B. The New Robust Optimization Model}

In the previous section, the robust optimization approach of [12] was discussed, and it was shown that this approach is not satisfactory for some problems with shared parameters. The previous section aimed to argue that in problems with shared uncertain parameters, the approaches which consider these parameters individually and ignores the dependency between them may lead to conflicts in their results. To the best of our knowledge, this situation has not been addressed by the previous studies in the robust optimization problems particularly when interval numbers express uncertain events.

To explain the new approach, it should be noted that in the linear optimization problems, the critical value of an uncertain parameter which causes the objective function to be worst is its upper bound or its lower bound. Therefore, we introduce two binary variables $\gamma_{1}$ and $\gamma_{2}$ for each uncertain parameter denoting whether the uncertain parameter takes its upper bound or its lower bound, respectively. Moreover, to explain the new approach, a maximization problem as following is considered:

$\operatorname{Max} z=C X$

$s . t$

$$
\begin{aligned}
& A X \leq b \\
& X \geq 0
\end{aligned}
$$

In this problem, $X$ is a $n \times 1$ vector of positive real variables, $C$ is $1 \times n$ a vector, $A$ is a $m \times n$ matrix, and $b$ is a $n \times 1$ vector of parameters. It is assumed that each entry $a_{i j}$ of the matrix $A$, each entry $c_{j}$ of vector $C$, and each entry $b_{i}$ of vector $b$ take values in the intervals $\left[a_{i j}-\hat{a}_{i j}, a_{i j}+\hat{a}_{i j}\right] \quad, \quad\left[c_{j}-\hat{c}_{j}, c_{j}+\hat{c}_{j}\right] \quad$ and $\left[b_{i}-\hat{b_{i}}, b_{i}+\hat{b_{i}}\right]$ respectively.

It also is assumed that there are $k$ uncertain events which could be shared between uncertain parameters. For each uncertain event $k$, two binary variables $\gamma_{1}^{k}$ and $\gamma_{2}^{k}$ are considered indicating whether that uncertain event appears in the robust solution with its upper bound or its lower bound. Moreover, we call $\gamma_{1}^{k}$ as the first robust binary variable of the uncertain event $k$, which will be equal to 1 if that event takes its upper bound in the robust solution or will be 0 otherwise. Similarly, $\gamma_{2}^{k}$ is called the second robust binary variable of the uncertain event $k$ and will be equal to 1 if that event appears with its lower bound in the robust solution or will be 0 otherwise. Each element of the model parameters is assumed to be related to only one uncertain event. In this regard, the notations $e^{c_{j}}, e^{a_{i j}}$ and $e^{b_{i}}$ are defined for indicating the related uncertain event of the element $c_{j}$, $a_{i j}$ and $b_{i}$ respectively. To distinguish the uncertain parameters which take their critical value from the ones that appear with their mean value in the robust solution, we define the matrix $A$, the vector $C$, and the vector $b$, which contain the elements $a_{i j}, c_{j}$ and $b_{j}$ respectively. Also, the matrix $\hat{A}$, the vector $\hat{C}$, and the vector $\hat{b}$ are introduced, which contain the elements $\hat{a}_{i j}, \hat{c}_{j}$ and $\hat{b}_{i}$ respectively. Remembering these notations, the matrix $\hat{A}^{\gamma_{1}}$, the vector $\hat{C}^{\gamma_{1}}$, and the vector $\hat{b}^{\gamma_{1}}$ are defined whose elements are respectively the corresponding elements of the matrix $\hat{A}$, $\hat{C}$ and $\hat{b}$ which are multiplied by the first robust binary variable of those elements. For example, the vector $\hat{C}^{\gamma_{1}}$ shows the vector whose $j^{\prime}$ th element is $\hat{c}_{j} \cdot \gamma_{1}^{e^{c_{j}}}$.

Similarly, the matrix $\hat{A}^{\gamma_{2}}$, the vector $\hat{C}^{\gamma_{2}}$, and the vector $\hat{b}^{\gamma / 2}$ are defined whose elements are respectively the corresponding elements of matrix $\hat{A}, \hat{C}$ and $\hat{b}$ which are multiplied by the second robust binary variable of those elements. Also, the parameter set $\Omega_{l}$ is defined as a set containing the uncertain parameters of type $l$ (for example, the parameters which are related to demand of products can 
be classified as the parameter set of demand). Taking into account the above notations, the proposed robust optimization model is presented as the following:

Model (1):

$$
\begin{aligned}
& \sum_{\substack{k \in \Omega_{j} \\
\gamma_{1}^{k}+\gamma_{2}^{k} \leq \Gamma_{l}, \forall l \\
\gamma_{1}^{k}+\gamma_{2}^{k} \leq 1 \\
\gamma_{1}^{k}, \gamma_{2}^{k} \in\{0,1\} \forall k \in K \\
\text { s.t. }}} \quad \operatorname{Max} z=\left(C-C_{1}^{\gamma_{1}}+C_{1}^{\gamma_{2}}\right) X \\
& \\
& \left(A-A_{1}^{\gamma_{1}}+A_{1}^{\gamma_{2}}\right) \cdot X \leq\left(b-b_{1}^{\gamma_{1}}+b_{1}^{\gamma_{1}}\right) \\
& X \geq 0
\end{aligned}
$$

In model (1) for given values of $\Gamma_{j} \mathrm{~s}$, the aim is to find a solution by selecting up to $\Gamma_{j}$ uncertain parameters in the set $\Omega_{j}$ and set the value of them equal to their upper bound or lower bound such that it leads to the worst optimal value of the objective function. This idea will cover the concepts of robustness in the proposed approach. Before explaining the solution approach proposed for converting this problem to an equivalent mixed-integer linear programming one, we claim such an approach is similar to [12] approach when there are no shared uncertain events in the problem. Actually, in [12] approach, the aim is to choose $\Gamma$ uncertain parameters in their critical value such that the solution space becomes the most restricted. In this way, the feasibility of the robust solution is ensured with high probability regardless of the realization of the uncertain events. Also, it is known from the operational research concepts that if the feasible space of the solutions becomes more restricted in an optimization problem, the value of the objective function will not be better, and it is possible to become worse. So the new approach results in a worst-case analysis regarding the sets $\Omega_{j}$ 's as the "uncertainty set". Hence, Lemma (1) is stated as below:

Lemma (1): the results of the new approach are the same as [12] approach when there are no shared parameters in the problem.

Now, the procedure used for solving Model (1) is described. Model (1) is a two-level combinatorial optimization model. In the first level, all combinations of first and second robust binary variables for all uncertain events are examined such that for each combination, the relations $\sum_{k \in \Omega_{l}} \gamma_{1}^{k}+\gamma_{2}^{k} \leq \Gamma_{l} \quad \forall l \quad, \quad \forall k, \gamma_{1}^{k}+\gamma_{2}^{k} \leq 1 \quad$ and $\gamma_{1}^{k}, \gamma_{2}^{k} \in\{0,1\} \forall k \in K$ are satisfied. In these relations, $K$ is the set of all uncertain events. Moreover, it should be pointed out that uncertain events in a solution could be considered in just one form of its upper bound, its lower bound, or its mean value. Therefore, the constraint $\gamma_{1}^{k}+\gamma_{1}^{k} \leq 1, \forall k$ should be held in the robust model.

Model (2):

$\operatorname{Max} z=\left(C-C_{1}^{\gamma_{1}}+C_{1}^{\gamma_{2}}\right) X$

s.t.

$$
\begin{aligned}
& \left(A-A_{1}^{\gamma_{1}}+A_{1}^{\gamma_{2}}\right) \cdot X \leq b-b_{1}^{\gamma_{1}}+b_{1}^{\gamma_{2}} \\
& X \geq 0
\end{aligned}
$$

Finally, each of the combinations that have the minimum value of the objective function $z$ is the robust solution of the proposed approach. Hence, for solving Model (2), the vector $\mathrm{X}$ acts as the variable of Model (2) and the values of $\gamma_{1}^{k}$ and $\gamma_{2}^{k}$ are regarded as known parameters. Now, to convert such nonlinear and combinatorial problem to a linear one, first, the Dual of Model (2) is considered as Model (3): Model (3)

$$
\begin{aligned}
& \operatorname{MinV}=w \cdot\left(b-b_{1}^{\gamma_{1}}+b_{1}^{\gamma_{2}}\right) \\
& \text { s.t. } \\
& \quad w \cdot\left(A-A_{1}^{\gamma_{1}}+A_{1}^{\gamma_{2}}\right) \geq\left(C-C_{1}^{\gamma_{1}}+C_{1}^{\gamma_{2}}\right) \\
& \quad w \geq 0
\end{aligned}
$$

It is known from the duality theorem that if a primal problem is bounded and feasible (as we suppose for our problem), then the dual problem is also bounded and has a finite solution, and these two solutions are equal. So we can substitute Model (2) with Model (3) into Model (1). This yields to Model (4) as follow:

Model (4)

$$
\begin{aligned}
& \underset{\substack{k \in \Omega_{l} \\
\gamma_{1}^{k}+\gamma_{2}^{k} \leq \Gamma_{l}, \forall l \\
\gamma_{1}^{k}+\gamma_{2}^{k} \leq 1 \forall k \in K \\
\gamma_{1}^{k}, \gamma_{2}^{k} \in\{0,1\} \forall k \in K}}{\operatorname{Min}} \quad \operatorname{Min} V=w \cdot\left(b-b_{1}^{\gamma_{1}}+b_{1}^{\gamma_{2}}\right) \\
& \text { s.t. } \\
& w .\left(A-A_{1}^{\gamma_{1}}+A_{1}^{\gamma_{2}}\right) \geq\left(C-C_{1}^{\gamma_{1}}+C_{1}^{\gamma_{2}}\right) \\
& w \geq 0
\end{aligned}
$$

The Min-Min model of Model (4) is equivalent to Model (5), which are shown as the following. This model is a mixed-integer non-linear model.

Model (5)

$$
\begin{aligned}
\operatorname{Min} V= & w \cdot\left(b-b_{1}^{\gamma_{1}}+b_{1}^{\gamma_{2}}\right) \\
\text { s.t. } & \\
& w \cdot\left(A-A_{1}^{\gamma_{1}}+A_{1}^{\gamma_{2}}\right) \geq\left(C-C_{1}^{\gamma_{1}}+C_{1}^{\gamma_{2}}\right) \\
& \sum_{k \in \Omega_{l}} \gamma_{1}^{k}+\gamma_{2}^{k} \leq \Gamma_{l} \forall l \\
& \gamma_{1}^{k}+\gamma_{2}^{k} \leq 1 \forall k \in K \\
& w \geq 0
\end{aligned}
$$

Until now, a min-max constraint two-level model has been converted to an equivalent one-level model. However, this new model is still nonlinear because in the following terms, the nonlinear terms exist:

$$
w \cdot\left(A-A_{1}^{\gamma_{1}}+A_{1}^{\gamma_{2}}\right) \geq\left(C-C_{1}^{\gamma_{1}}+C_{1}^{\gamma_{2}}\right) ; w \cdot\left(b-b_{1}^{\gamma_{1}}+b_{1}^{\gamma_{2}}\right) .
$$

Also, the nonlinear terms

$$
w \cdot\left(A-A_{1}^{\gamma_{1}}+A_{1}^{\gamma_{2}}\right) \geq\left(C-C_{1}^{\gamma_{1}}+C_{1}^{\gamma_{2}}\right)
$$


are the consequence of multiplying the vector $\mathrm{w}$ by matrix $A_{1}^{\gamma_{1}}$ or $A_{2}^{\gamma_{2}}$. To get rid of such a nonlinearity term, a simple conversation could be used. Indeed, the nonlinear terms appeared in the forms of:

$w_{1} \cdot a_{1 j} \cdot \gamma_{1}^{a_{1 j}}, \ldots, w_{n} \cdot a_{n j} \cdot \gamma_{1}^{a_{n j}}, w_{1} \cdot a_{1 j} \cdot \gamma_{2}^{a_{1 j}}, \ldots, w_{n} \cdot a_{n j} \cdot \gamma_{2}^{a_{2 j}}$,

and

$w_{1} b_{1} \cdot \gamma_{1}^{b_{1}}, \ldots, w_{n} b_{n} \cdot \gamma_{1}^{b_{n}}, w_{1} b_{1} \cdot \gamma_{2}^{b_{1}}, \ldots, w_{n} b_{n} \cdot \gamma_{1}^{b_{n}}$. Therefore,

each nonlinear term composed of a positive variable multiplied by a binary variable. To linearize such non-linear terms, if we consider a term $y . z$ in which $y$ is a positive variable and $z$ is a binary variable, then the term $y . z$ could be replaced by the new variable $u$ for which the following constraint is exact:

$$
\begin{gathered}
u=y \cdot z \\
y-M(1-z) \leq u \leq y+M(1-z) \\
u \leq M . z
\end{gathered}
$$

Therefore, all nonlinear terms in Model (5) are converted to linear ones using the constraint (40), (41) and (42) and the resulting model is a mixed-integer linear programming model that covers the idea of robustness in the proposed approach.

\section{Model Validation}

As we claimed before, the proposed approach results in robust solutions similar to Bertsimas and Sim's approach particularly when there are no repeated or shared events in the model constraints. This could be examined by considering the following problem:

Problem (2):

$$
\begin{gathered}
\max Z=2 x_{1}+3 x_{2}-2 x_{3}+x_{4} \\
\text { s.t. } \\
\tilde{a_{1}} \cdot x_{1}+\tilde{a}_{2} \cdot x_{2}+\tilde{a}_{3} \cdot x_{3}+\tilde{a}_{4} \cdot x_{4} \leq 50 \\
b_{1} \cdot x_{1}+b_{2} \cdot x_{2}+b_{3} \cdot x_{3}+b_{4} \cdot x_{4} \leq 60 \\
0 \leq x_{1}, x_{2}, x_{3}, x_{4} \leq 2
\end{gathered}
$$

The value of the uncertain parameters in Problem (2) is considered as Table IV:

TABLE IV. The VALue of THE PARAmeters IN PROBLEM (2)

\begin{tabular}{|c|llll|}
\hline $\boldsymbol{i}$ & $\mathbf{1}$ & $\mathbf{2}$ & $\mathbf{3}$ & $\mathbf{4}$ \\
\hline $\boldsymbol{a}_{\boldsymbol{i}}$ & 8 & 5 & 6 & 7 \\
$\widehat{\boldsymbol{a}}_{\bar{i}}$ & 2 & 4 & 3 & 5 \\
$\boldsymbol{b}_{\boldsymbol{i}}$ & 6 & 4 & 8 & 7 \\
$\widehat{\boldsymbol{b}}_{\tilde{i}}$ & 3 & 2 & 4 & 6 \\
\hline
\end{tabular}

Considering $P_{1 \mathrm{i}}$ and $P_{2 \mathrm{i}}$ as the related variables of the uncertain parameters in the constraints (44) and (45) and solving this model using [12] approach, the following results as TableV will be obtained:

TABLE V. Results of the PROBLEM (2) USING Bertsimas AND SIM (2004) APPROACH

\begin{tabular}{|c|c|c|c|c|}
\hline$\Gamma_{1}, \Gamma_{2}$ & 1 & 2 & 3 & 4 \\
\hline $\mathrm{Z}$ & 12 & 11.33 & 11 & 11 \\
\hline$P_{1 i}>0$ & & $P_{12}, P_{14}$, & $P_{11}, P_{12}$, & $P_{11}, P_{12}, P_{13}$, \\
and & $P_{14,} P_{24}$ & $P_{21}, P_{22}$ & $P_{14}, P_{21}, P_{22}$, & $P_{14} P_{21}, P_{22}$, \\
$P_{2 i}>0$ & & $P_{23}$ & $P_{23}, P_{24}$ \\
\hline
\end{tabular}

The results of Table $\mathrm{V}$ indicate that in the optimal robust solutions of Problem (2), when $\Gamma_{1}=\Gamma_{2}=1$, the parameters $a_{4}$ and $b_{4}$ are in their critical values, when $\Gamma_{1}=\Gamma_{2}=2, a_{2}$, $a_{4}, b_{1}$ and $b_{2}$ are in their critical values and so on.

This problem could also be solved by the proposed approach considering the following model:

$$
\begin{aligned}
& \text { Min } V=50 w_{1}+60 w_{2}+2 w_{3}+2 w_{4}+2 w_{5}+2 w_{6} \\
& \text { s.t. } \\
& \left(w_{1}, w_{2}, w_{3}, w_{4}, w_{5}, w_{6}\right)(A-B+C) \geq\left(\begin{array}{llll}
2 & 3 & -2 & 1
\end{array}\right)
\end{aligned}
$$

$$
\begin{gathered}
A=\left(\begin{array}{llll}
8 & 5 & 6 & 7 \\
6 & 4 & 8 & 7 \\
1 & 0 & 0 & 0 \\
0 & 1 & 0 & 0 \\
0 & 0 & 1 & 0 \\
0 & 0 & 0 & 1
\end{array}\right), B=\left(\begin{array}{cccc}
2 \gamma_{1}^{1} & 4 \gamma_{1}^{2} & 3 \gamma_{1}^{3} & 5 \gamma_{1}^{4} \\
3 \gamma_{1}^{5} & 2 \gamma_{1}^{6} & 4 \gamma_{1}^{7} & 6 \gamma_{1}^{8} \\
0 & 0 & 0 & 0 \\
0 & 0 & 0 & 0 \\
0 & 0 & 0 & 0 \\
0 & 0 & 0 & 0
\end{array}\right), \\
C=\left(\begin{array}{cccc}
2 \gamma_{2}^{1} & 4 \gamma_{2}^{2} & 3 \gamma_{2}^{3} & 5 \gamma_{2}^{4} \\
3 \gamma_{2}^{5} & 2 \gamma_{2}^{6} & 4 \gamma_{2}^{7} & 4 \gamma_{2}^{8} \\
0 & 0 & 0 & 0 \\
0 & 0 & 0 \\
0 & 0 \\
0 & 0 \\
0 & 0
\end{array}\right) \\
\gamma_{1}^{k}+\gamma_{1}^{k} \leq 1 \\
\gamma_{2}^{k}+\gamma_{2}^{k} \leq 1 \\
\sum_{k \in\{1,2,3,4\}} \gamma_{1}^{k}+\gamma_{2}^{k}=\Gamma_{1} \\
\sum_{k \in\{5,6,7,8\}} \gamma_{1}^{k}+\gamma_{2}^{k}=\Gamma_{2} \\
\gamma_{1}^{k}, \gamma_{2}^{k}=\{0,1\} \quad \forall k \in\{1,2,3,4,5,6,7,8\} \\
\left(w_{1}, w_{2}, w_{3}, w_{4}, w_{5}, w_{6}\right) \geq 0
\end{gathered}
$$

In this model, we have nonlinear terms $w_{k} \cdot \gamma_{1}^{k}, w_{k} \cdot \gamma_{2}^{k} \quad \forall k=\{1,2,3,4,5,6,7,8\}$. For the above terms, the variables $z_{1}^{k}=w_{k} \cdot \gamma_{1}^{k}, z_{2}^{k}=w_{k} \cdot \gamma_{2}^{k}$ are defined, and the constraints (55) - (58) are added to the above model for the sake of linearization: 


$$
\begin{array}{lr}
w_{k}-M\left(1-\gamma_{1}^{k}\right) \leq z_{1}^{k} \leq w_{k}+M\left(1-\gamma_{1}^{k}\right) & \forall k \in\{1,2,3,4,5,6,7,8\} \\
w_{k}-M\left(1-\gamma_{2}^{k}\right) \leq z_{2}^{k} \leq w_{k}+M\left(1-\gamma_{2}^{k}\right) & \forall k \in\{1,2,3,4,5,6,7,8\} \\
z_{1}^{k} \leq M \cdot \gamma_{1}^{k} \quad \forall k=\{1,2,3,4,5,6,7,8\} & \\
z_{2}^{k} \leq M \cdot \gamma_{2}^{k} & \forall k=\{1,2,3,4,5,6,7,8\}
\end{array}
$$

Solving Problem (2) by the proposed approach yields the results as Table VI.

TABLE VI. Results of Problem (2) Using the Proposed Approach

\begin{tabular}{|c|c|c|c|c|}
\hline$\Gamma_{1}, \Gamma_{2}$ & 1 & 2 & 3 & 4 \\
\hline $\mathrm{V}$ & 12 & 11.33 & 11 & 11 \\
\hline$\gamma_{u}^{k} \mid \gamma_{u}^{k}=1$ & $\gamma_{2}^{4}, \gamma_{1}^{8}$ & $\gamma_{2}^{2}, \gamma_{1}^{4}$ & $\gamma_{2}^{1}, \gamma_{2}^{2}, \gamma_{2}^{4}$ & $\gamma_{1}^{2}, \gamma_{2}^{2}, \gamma_{1}^{3}$ \\
$u=1,2 k=1$, \\
& & $\gamma_{2}^{4}, 3,4$ & $\gamma_{1}^{5}, \gamma_{2}^{6}, \gamma_{2}^{7}$ & $\gamma_{1}^{5}, \gamma_{2}^{6}, \gamma_{1}^{7}$ \\
& & & & \\
\hline
\end{tabular}

It is seen in Table VI that the results of the proposed approach are the same as [12] approach. For example, in both approaches, when $\Gamma_{1}=\Gamma_{2}=2$ the uncertain parameters $a_{2}, a_{4}, b_{1}$ and $b_{2}$ are appeared with their critical value in the optimal robust solution. Moreover, the objective function value of both approaches is the same for different values of $\Gamma_{1}$ and $\Gamma_{2}$. However, this problem has not any shared events. To show the validity of the model for the problem with the shared uncertain events, we come back to Problem (1) and show how the proposed approach could solve the challenges of inconsistency addressed in that problem.

In Problem (1), the parameters $d_{i}$ 's were uncertain. For each $d_{i}$, two binary variables $\gamma_{1}^{i}$ and $\gamma_{2}^{i}$ could be defined that has a description as before. Using Model (5), the robust model of Problem (1) will be as follow:

$$
\begin{aligned}
& \text { Min } \quad V=C w_{1}+w_{2}+w_{3}+w_{4}+w_{5} \\
& \text { s.t. } \\
& \left(w_{1}, w_{2}, w_{3}, w_{4}, w_{5}\right) \cdot(A-B+C) \geq\left(c_{1} \cdot d_{1}-c_{1} \cdot \hat{d}_{1} \cdot \gamma_{1}^{1}+c_{1} \hat{d}_{1} \cdot \gamma_{2}^{1},\right. \\
& c_{2} \cdot d_{2}-c_{2} \cdot \hat{d}_{2} \cdot \gamma_{1}^{2}+c_{2} \cdot \hat{d}_{2} \cdot \gamma_{2}^{2}, c_{3} \cdot d_{3}-c_{3} \cdot \hat{d}_{3} \cdot \gamma_{1}^{3}+c_{3} \cdot \hat{d}_{3} \cdot \gamma_{2}^{3}, \\
& \left.c_{4} \cdot d_{4}-c_{4} \cdot \hat{d}_{4} \cdot \gamma_{1}^{4}+c_{4} \cdot \hat{d}_{4} \cdot \gamma_{2}^{4}\right) \\
& \text { where: } \\
& \begin{aligned}
A & =\left(\begin{array}{cccc}
a_{1} \cdot d_{1} & a_{2} \cdot d_{2} & a_{3} \cdot d_{3} & a_{4} \cdot d_{4} \\
1 & 0 & 0 & 0 \\
0 & 1 & 0 & 0 \\
0 & 0 & 1 & 0 \\
0 & 0 & 0 & 1
\end{array}\right) \\
B & =\left(\begin{array}{cccc}
a_{1} \cdot \hat{d}_{1} \cdot \gamma_{1}^{1} & a_{2} \cdot \hat{d}_{2} \cdot \gamma_{1}^{2} & a_{3} \cdot \hat{d}_{3} \cdot \gamma_{1}^{3} & a_{4} \cdot \hat{d}_{4} \cdot \gamma_{1}^{4} \\
0 & 0 & 0 & 0 \\
0 & 0 & 0 & 0 \\
0 & 0 & 0 & 0 \\
0 & 0 & 0 & 0
\end{array}\right) \\
C & =\left(\begin{array}{cccc}
a_{1} \cdot \hat{d}_{1} \cdot \gamma_{2}^{2} & a_{2} \cdot \hat{d}_{2} \cdot \gamma_{2}^{2} & a_{3} \cdot \hat{d}_{3} \cdot \gamma_{2}^{3} & a_{4} \cdot \hat{d}_{4} \cdot \gamma_{2}^{4} \\
0 & 0 & 0 & 0 \\
0 & 0 & 0 & 0 \\
0 & 0 & 0 & 0 \\
0 & 0 & 0 & 0
\end{array}\right)
\end{aligned}
\end{aligned}
$$

$$
\begin{gathered}
\sum_{j \in\{1,2,3,4\}} \gamma_{1}^{k}+\gamma_{2}^{k} \leq \Gamma \\
w_{j} \geq 0 \quad \forall j=\{1,2,3,4,5\}, \quad \gamma_{1}^{k}, \gamma_{1}^{k}=\{0,1\} \\
\forall k=\{1,2,3,4\}
\end{gathered}
$$

Using the data in Table (1) and solving the shown model in (59) - (62), the results for different value of $\Gamma$ will be as Table VII:

TABLE VII. Results of Problem (1) Using the Proposed Approach

\begin{tabular}{|c|c|c|c|c|}
\hline$\Gamma$ & 1 & 2 & 3 & 4 \\
\hline $\mathbf{V}$ & 112 & 97 & 85 & 77 \\
\hline$\gamma_{i}^{1}=\mathbf{1}$ & $\gamma_{4}^{1}$ & $\gamma_{1}^{1}, \gamma_{4}^{1}$ & $\gamma_{1}^{1}, \gamma_{3}^{1}, \gamma_{4}^{1}$ & $\gamma_{1}^{1}, \gamma_{2}^{1}, \gamma_{3}^{1}, \gamma_{4}^{1}$ \\
\hline
\end{tabular}

As the results in Table VI indicate when $\Gamma=2$ the objective function will be equal to 104 and the variable $\gamma_{1}^{1}$ and $\gamma_{1}^{4}$ will take value equal to one in the optimal solution which indicates the parameters $d_{1}$ and $d_{4}$ in the robust solution will take their lower bound values. It is clear that the value of the objective function has been improved using the new method. This can be explained by the fact that in [12] approach, $\Gamma$ uncertain parameters considered by their critical values in both the objective function and the constraint. However, because the objective function and the constraint in Problem (1) are dependent, some parameters value that makes the objective function the worst may lead to a better feasible space for the variables and, in turn, make eligible points feasible.

\section{DISCUSSION}

In this section, some applications of the proposed approach in the real world problems are discussed. The first regarded example is the study of [35]. They considered a two objective model for minimizing the cost and risk of transportation in a network of hazard material supply chain. In that article, the risk was measured as the sum of risks in all paths of the network. Also, a threshold for acceptable risk of each path was considered. In that study, if the risk per shipment is considered as an uncertain parameter, it is clear that this parameter exists in both objective function and the constraints related to the maximum allowed risk of each path. The proposed robust approach suggested in this paper apply to this problem. So, the first application of the proposed approach is for the problems in which some limitations exist on the objective functions as the model constraints. Minimizing the cost of investment by imposing a limitation on the budgets of different investment areas is another example of this type [36].

The demand for products and services plays a vital role in business models. This parameter takes various forms in mathematical models of problems. In some problems, it affects variables such as the number of vehicles, the responded demands of customers, the reached level of quality or reliability, etc. Also, it may appear as a right-hand side parameter in the model constraints [37]. When the latter 
is the case, it should be noted that there is only one such uncertain parameter in a constraint - so defining the level of protection against uncertainty as $\Gamma$ (the parameters related to the confidence level in [12] in a constraint with just one uncertain parameter is meaningless. Actually, in this case, this parameter can be regarded as a critical parameter or not. As we mentioned in section (3), the concept of "uncertainty $s e t$ " can be more interpretable in this case. For example, the "demand set" could be regarded as a set that includes all customers' demand for products. Hence, by labeling these parameters as binary variables and considering the protection level $\Gamma$ for the parameters in the demand set, the concept of robustness and protection level against uncertainty is seemed to be more desirable and applicable. Another example of the "uncertainty set" is the processing time of works on machines in the job shop and flow shop scheduling problems. This parameter might be uncertain and shared between the model constraints [38]. Again, by defining "processing time set," the concept of robustness can be extended to these problems. The project scheduling problem (PSP) could be considered [39] as another type of problem with common uncertain parameters. In this problem, the duration of activities in a project network can be regarded as a shared uncertain parameter. In the precedence constraints of this problem, only one duration time exists. Also, the duration time of an activity is shared between the precedence constraints of all its processors. Therefore, in this problem, "activity duration set" could be defined and the protection against uncertainty could be considered for this set of parameters.

\section{CONCLUSION}

In this research, a new robust optimization approach has been introduced for the problems with uncertain parameters in which these parameters are shared between the model constraints and objective function. The situations in which this model is useful were discussed, and the model validity was shown using numerical examples. It was shown that the suggested Min-Max strategy could prepare less conservative solutions than a fully conservative approach. It was also shown that this procedure yields the results the same as [12] approach when there is no shared parameter in a problem. However, in the problems with shared uncertain parameters, [12] had some inconsistencies, which can be fixed by the proposed approach. Also, we utilized the concept of "uncertainty set" that extends the concept of robustness to the sets with the same type of parameters. For future research, the implementation of the proposed approach for real-world problems is suggested.

\section{REFERENCES}

[1] Gabrel, V., C. Murat, and A. Thiele, Recent advances in robust optimization: An overview. European journal of operational research, 2014. 235(3): p. 471-483.

[2] Wang, F., X. Lai, and N. Shi, A multi-objective optimization for green supply chain network design. Decision support systems, 2011. 51(2): p. $262-269$.
[3] Yuan, Y., Z. Li, and B. Huang, Robust optimization approximation for joint chance constrained optimization problem. Journal of Global Optimization, 2017. 67(4): p. 805-827.

[4] Chen, W., et al., A procedure for robust design: minimizing variations caused by noise factors and control factors. 1996.

[5] Jeyakumar, V. and G. Li, Robust solutions of quadratic optimization over single quadratic constraint under interval uncertainty. Journal of Global Optimization, 2013. 55(2): p. 209-226.

[6] Ben-Tal, A. and A. Nemirovski, Selected topics in robust convex optimization. Mathematical Programming, 2008. 112(1): p. 125-158.

[7] Bertsimas, D., D.B. Brown, and C. Caramanis, Theory and applications of robust optimization. SIAM review, 2011. 53(3): p. 464-501.

[8] Thiele, A., A note on issues of over-conservatism in robust optimization with cost uncertainty. Optimization, 2010. 59(7): p. 1033-1040.

[9] Gancarova, M. and M.J. Todd, A robust robust optimization result. Operations research letters, 2012. 40(1): p. 2-5.

[10] $\mathrm{Hu}, \mathrm{J}$. and S. Mehrotra, Robust and stochastically weighted multiobjective optimization models and reformulations. Operations research, 2012. 60(4): p. 936-953.

[11] Roy, B., Robustness in operational research and decision aiding: A multi-faceted issue. European Journal of Operational Research, 2010. 200(3): p. 629-638.

[12] Bertsimas, D. and M. Sim, The price of robustness. Operations research, 2004. 52(1): p. 35-53.

[13] Gorissen, B.L., et al., Deriving robust and globalized robust solutions of uncertain linear programs with general convex uncertainty sets. Operations Research, 2014. 62(3): p. 672-679.

[14] Beck, A. and A. Ben-Tal, Duality in robust optimization: primal worst equals dual best. Operations Research Letters, 2009. 37(1): p. $1-6$.

[15] Taguchi, G., Introduction to quality engineering: designing quality into products and processes. 1986.

[16] Mulvey, J.M., R.J. Vanderbei, and S.A. Zenios, Robust optimization of large-scale systems. Operations research, 1995. 43(2): p. 264-281.

[17] Soyster, A.L., Convex programming with set-inclusive constraints and applications to inexact linear programming. Operations research, 1973. 21(5): p. 1154-1157.

[18] Kang, S.-C., Robust linear optimization using distributional information. 2008: ProQuest.

[19] Doan, X.V., Optimization under moment, robust, and data-driven models of uncertainty. 2010, Massachusetts Institute of Technology.

[20] Assavapokee, T., M.J. Realff, and J.C. Ammons, Min-max regret robust optimization approach on interval data uncertainty. Journal of Optimization Theory and Applications, 2008. 137(2): p. 297-316.

[21] Assavapokee, T., et al., Scenario relaxation algorithm for finite scenario-based min-max regret and min-max relative regret robust optimization. Computers \& operations research, 2008. 35(6): p. 20932102.

[22] Chen, X. and Y. Zhang, Uncertain linear programs: Extended affinely adjustable robust counterparts. Operations Research, 2009. 57(6): p. 1469-1482.

[23] Bertsimas, D., D.A. Iancu, and P.A. Parrilo, Optimality of affine policies in multistage robust optimization. Mathematics of Operations Research, 2010. 35(2): p. 363-394.

[24] Chen, X., M. Sim, and P. Sun, A robust optimization perspective on stochastic programming. Operations Research, 2007. 55(6): p. 10581071.

[25] Dentcheva, D. and A. Ruszczyński, Robust stochastic dominance and its application to risk-averse optimization. Mathematical Programming, 2010. 123(1): p. 85.

[26] Bertsimas, D. and A. Takeda, Optimizing over coherent risk measures and non-convexities: a robust mixed integer optimization approach. 
Computational Optimization and Applications, 2015. 62(3): p. 613639.

[27] Alberto Campos, F., J. Villar, and M. Jimenez, Robust solutions using fuzzy chance constraints. Engineering Optimization, 2006. 38(6): p. 627-645.

[28] Ben-Tal, A. and A. Nemirovski, Robust convex optimization. Mathematics of operations research, 1998. 23(4): p. 769-805.

[29] Beyer, H.-G., M. Olhofer, and B. Sendhoff. On the Behavior of $(\mu / \mu 1$, ג)-ES Optimizing Functions Disturbed by Generalized Noise. in FOGA. 2002.

[30] Das, I., Robustness optimization for constrained nonlinear programming problems. Engineering Optimization+ A35, 2000. 32(5): p. 585-618.

[31] Birge, J.R. and F. Louveaux, Introduction to stochastic programming. 2011: Springer Science \& Business Media.

[32] El Ghaoui, L. and H. Lebret, Robust solutions to least-squares problems with uncertain data. SIAM Journal on matrix analysis and applications, 1997. 18(4): p. 1035-1064.

[33] Medeiros, D., et al., Proceedings of the 30th conference on Winter simulation. 1998, IEEE Computer Society Press.
[34] Ben-Tal, A. and A. Nemirovski, Robust solutions of linear programming problems contaminated with uncertain data. Mathematical programming, 2000. 88(3): p. 411-424.

[35] Xie, Y., et al., A multimodal location and routing model for hazardous materials transportation. Journal of hazardous materials, 2012. 227: p. 135-141.

[36] Nickel, S., F. Saldanha-da-Gama, and H.-P. Ziegler, A multi-stage stochastic supply network design problem with financial decisions and risk management. Omega, 2012. 40(5): p. 511-524.

[37] Zhu, J., et al. Resources allocation problem for local reserve depots in disaster management based on scenario analysis. in The 7th International symposium on operations research and its applications. Lijiang, China. 2008.

[38] Šeda, M., Mathematical models of flow shop and job shop scheduling problems. World Academy of Science, Engineering and Technology, 2007. 1(31): p. 122-127.

[39] Brucker, P., et al., A branch and bound algorithm for the resourceconstrained project scheduling problem. European journal of operational research, 1998. 107(2): p. 272-288. 